\title{
Multilagen-Mikrofluidikplattform für die Zellkultivierung mit integrierter Dickschichtsensorik
}

\author{
Mathias Busek ${ }^{1}$, Stefan Grünzner ${ }^{1}$, Volker Franke ${ }^{1}$, Frank Sonntag ${ }^{1}$ \\ ${ }^{1}$ Fraunhofer IWS Dresden, Winterbergstraße 28, 01277 Dresden
}

\begin{abstract}
Zusammenfassung
Beschrieben wird eine Technologiekette zur Integration von elektrochemischen bzw. elektrischen Dickschichtsensoren in eine mehrlagige Mikrofluidikplattform für die Zellkultivierung. Dafür wurde ein kommerziell erhältlicher Biosensor mit einer Clark-Elektrode auf Aluminiumoxid-Substrat direkt in den Polymerverbund einlaminiert. Die Ermittlung der optimalen Fügeparameter erfolgte dabei zuerst an verschiedenen Teststrukturen und anschließende Dichtigkeitsprüfung mit Indikatorflüssigkeit. Durch gezielte Laserbearbeitung der Substrate konnte eine verbesserte Verbindung zwischen Keramik und Polymer erreicht werden. Im Anschluss erfolgte die Integration des beschriebenen Sensors in eine Durchflussfluidik. Schließlich konnte durch die erfolgreiche amperometrische Sauerstoffmessung in verschiedenen Testflüssigkeiten die Funktionalität des Sensors auch nach der Lamination nachgewiesen werden. In nachfolgenden Arbeiten kann durch die Integration einer Membran die Trennung von Elektrolyt und Messgut erfolgen. Mit der entwickelten Prozesskette ist überdies der Grundstein für die erfolgreiche Implementierung weiterer Sensoren z.B. zur Messung des transepithelialen elektrischen Widerstands (TEER) gelegt.
\end{abstract}

Keywords: Mikrofluidik, Lab-on-a-Chip, Sensorintegration, Zellkultur, elektrochemische Sensorik.

\section{Einleitung}

Mikrofluidische oder auch Lab-on-a-Chip Systeme finden zunehmend Anwendung in der Biotechnologie. Einsatzmöglichkeiten finden sich z.B. beim Nachweis spezifischer Biomarker [1] oder bei der Kultivierung humaner Zellen [2]. Letzteres ist von besonderem Interesse, weil Systeme zur Mikroperfusion weitaus höhere Zelldichten ermöglichen und die in-vivo Situation besser nachbilden als statische Zellkultursysteme [3]. Ein aktueller Trend bei der Entwicklung neuer Lab-on-a-Chip Systeme für die Zellkultivierung bilden dabei sogenannte Body-on-a-Chip Systeme, welche verschiedene Gewebemodelle in einem gemeinsamen Kreislauf kultivieren [4]. Dieser neuartige Ansatz ermöglicht es die Interaktion verschiedener Gewebetypen auf dem Chip nachzustellen und bietet die Möglichkeit Tierversuche zukünftig zu ersetzen. Dies ist insbesondere deswegen von Interesse, weil Tierversuchsergebnisse in der pharmazeutischen Wirkstofftestung immer schwerer auf die Exposition im Menschen übertragbar sind [5]. Ein solches mikrofluidisches System ist in den letzten Jahren entwickelt worden [6] und beinhaltet neben einer Mikropumpe, verschiedene Reservoire und Ventile auch zwei Zellkulturkammern. In diesen Bereichen konnten erfolgreich verschiedene Organoide gezüchtet werden, z.B. Leber [7], Haut [8] oder Niere [9]. Die
Vitalität der kultivierten Zellen sowie verschiedene weitere Prozessparameter wie z.B. die Sauerstoffkonzentration werden optisch gemessen [10]. Da die mikrofluidische Plattform neuerdings durch das Laminieren von laserstrukturierten Polymerfolien hergestellt wird [11] ist zukünftig auch die Integration von elektrochemischen Sensoren denkbar. Neben der amperometrischen Messung des Sauerstoff- oder Glukosegehalts [12] ist so z.B. eine TEER-Messung zur Charakterisierung der Zelldichte möglich [13]. Vor Allem die Sauerstoffmessung ist von besonderem Interesse, da eine SauerstoffUnterversorgung (Hypoxie) bei Zellen spezielle Prozesse auslöst, welche im Zusammenhang mit Tumorwachstum [14] und Entzündungsprozessen stehen [16]. Auf die beschriebene Zellkulturplattform übertragen, ist durch die Integration von Sauerstoffsensoren eine automatisierte Reglung der Sauerstoffkonzentration möglich [17]. Somit können Hypoxie-Untersuchungen an Zellkulturen automatisiert und reproduzierbar durchgeführt werden.

\section{Material und Methoden}

Die Herstellung des mikrofluidischen Systems erfolgt durch das thermische Fügen von laserstrukturierten Polycarbonatfolien [15]. Dadurch können komplexe Strukturen über mehrere Ebenen hergestellt werden. Im ersten Schritt werden die Konturen der einzelnen 
Lagen abgeleitet und dann mit einer Lasermikrostrukturierungsanlage (microSTRUCT vario, 3D-MICROMAC) mit Pikosekundenlaser (FUEGO, TIME-BANDWIDTH PRODUCTS) in die Folien geschnitten. Im Anschluss werden die einzelnen Lagen mit Hilfe einer Heißpresse thermisch gefügt. Fluidische Aktoren wie Pumpen oder Ventile wurden in das System integriert indem eine Elastomerfolie in den Verbund über einen nasschemischen Prozess eingebracht wurde [18]. In Abb. 1 ist die Explosionsdarstellung eines mikrofluidischen Systems dargestellt.

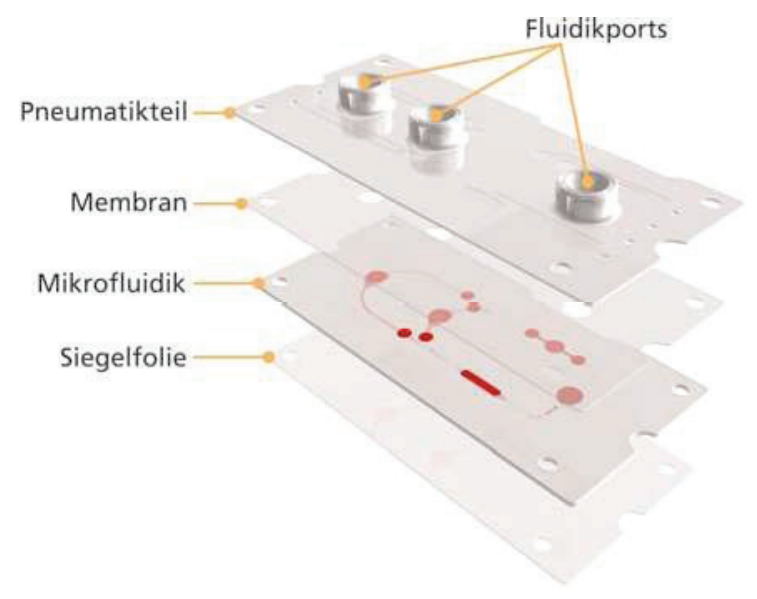

Abb. 1: Explosionsdarstellung eines mikrofluidischen Multilagensystems mit pneumatischem Teil, der biegsamen Membran und dem fluidischen Teil.

Erkennbar ist auf der Oberseite den Pneumatikteil, mit Kanälen welche die Druckluft zu den integrierten Aktoren weiterleiten sowie verschiedene pneumatische und fluidische Konnektoren. Zwischen der Mikrofluidik, welche die Kanäle und Zellkulturkammern enthält, befindet sich die elastische Membran. Eine Siegelfolie auf der Unterseite sorgt schließlich für den fluidischdichten Abschluss und einen guten optischen Zugang. Das gesamte System kann in einem Support fixiert werden, welcher neben der pneumatischen Kontaktierung auch die Temperierung des Zellkultursystems übernimmt. Eine spezielle pneumatische Ansteuereinheit mit 24 Ausgängen ermöglicht es die eingebrachten Membranen zu aktuieren und heizt die Supports auf $37^{\circ} \mathrm{C}$. Die integrierte Pumpe wurde dabei ausführlich mittels Micro-Particle-Image-Velocimetry ( $\mu$ PIV) [19] charakterisiert und ein entsprechendes mathematisches Modell abgeleitet [20]. Zudem sind die Integration weiterer Funktionselemente wie ein Oxygenator oder sauerstoffsensitive Farbstoffe [21] denkbar.

Neben optischer Sensorik gibt es eine Vielzahl elektrischer und elektrochemischer Sensoren
z.B. zur Überwachung des Sauerstoffgehalts, $\mathrm{PH}-$ Werts oder der Laktat-Konzentration [22] im Medium. Viele sind dabei in Dickschichttechnik hergestellt. Dabei werden Leiterzüge und Elektrodenmaterialien in Form von Pasten mit einem Siebdruckverfahren auf ein Substrat (z.B. Aluminiumoxid) gedruckt und anschließend bei hohen Temperaturen ausgebrannt [23]. Der Vorteil solcher Sensoren liegt in ihrer Temperatur und Chemikalienbeständigkeit. Die Bioverträglichkeit wird gewährleistet, indem der Sensor mit einer Membran vom Kulturmedium getrennt wird [24]. Bestimmte Kenngrößen wie der transepitheliale elektrische Widerstand (TEER) lassen sich überdies nur elektrochemisch und damit invasiv messen [13]. Es soll ein kommerzieller Dickschichtsensor (Siehe Abb. 2) zur elektrochemischen Messung der Sauerstoffkonzentration (Pt-Pt-Ag-Elektrode, BST BIO SENSOR TECHNOLOGY GMBH) in die beschriebene Mikrofluidikplattform integriert werden.

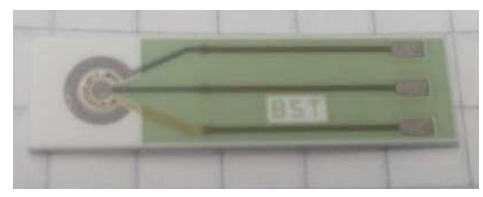

Abb. 2: Clark-Elektrode für die Sauerstoffmessung, gedruckt auf $\mathrm{Al}_{2} \mathrm{O}_{3}-$ Substrat.

Anschließend soll der Sauerstoffgehalt im Kulturmedium gemessen werden. Diese Sauerstoffmessung beruht auf dem von Clark vorgestellten Prinzip [25]. An der Pt-Elektrode findet bei Anlegen einer Polarisationsspannung (ca. -0.7 V) im Elektrolyten (üblicherweise gesättigte $\mathrm{KCl}$ - Lösung) folgende elektrochemische Reaktion statt:

$\mathrm{O}_{2}+4 e^{-}+2 \mathrm{H}_{2} \mathrm{O} \rightarrow 4 \mathrm{OH}^{-}$

In Folge dieser Reaktion fließt ein Strom, welcher direkt zu dem Sauerstoffpartialdruck $p_{\mathrm{O}_{2}}$ im Elektrolyten proportional ist. Die Lebensdauer des Sensors kann erhöht werden sowie unerwünschte Ablagerungen an den Elektroden können verhindert werden, indem der Sensor mit einer sauerstoffpermeablen Membran vom Messgut getrennt wird. Der zu messende Sauerstoff gelangt dann über Diffusionsprozesse aus dem Probenraum zum Sensor. Der Einfachheit halber wurde diese Membran vorerst weggelassen und das Zellkulturmedium direkt als Elektrolyt verwendet. Das Auslesen des Sensors übernimmt das Potentiostat Emstat von der Firma PALMSENS. Dieses Gerät ermöglicht verschiedene Betriebsarten u.a. das Aufnehmen eines Voltammograms oder die amperometrische Messung. 


\section{Sensorintegration}

Der beschriebene Sensor besitzt eine Länge von $25,5 \mathrm{~mm}$ und eine Breite von $7 \mathrm{~mm}$ und wurde auf ein $0.65 \mathrm{~mm}$ starkes AluminiumoxidSubstrat gedruckt. Die elektrischen Anschlüsse befinden sich Gegenüber zu Arbeits- und Referenzelektrode und sollten bei der Integration möglichst zugängig sein. Weiterhin sollte der Sensor möglichst direkt in die Fluidik integriert werden und nur ein geringes Totvolumen aufweisen. Eine Integration des Sensors ist prinzipiell auf unterschiedliche Weise möglich:

1. Einkleben.

2. Einlaminieren.

3. Eingießen.

4. Selektives Aufschmelzen

5. Lösungsmittel-basiertes Fügen

6. Plasmafügen.

Viele der genannten Verfahren lassen sich mit dem Keramiksubstrat nicht oder nur schlecht umsetzen, weswegen vorerst das direkte Einlaminieren des Sensors in den Polymerverbund angewandt wurde.

Dafür wurden zuerst runde $\mathrm{Al}_{2} \mathrm{O}_{3}$ Plättchen mit einem Durchmesser von $22 \mathrm{~mm}$ und einer Dicke von $250 \mu \mathrm{m}$ als Sensor-Dummys verwendet, um die idealen Prozessparameter für den Laminationsprozess zu finden. Diese wurden in passgenauen Taschen im Mikrofluidik-System eingelegt und das Gesamtsystem wie in [11] beschrieben thermisch gefügt. Im Anschluss wurde die Dichtigkeit der Fügeverbindung durch Aufbringung einer gefärbten Flüssigkeit nachgebildet. In Abb. 3 ist eine solche Teststruktur abgebildet.

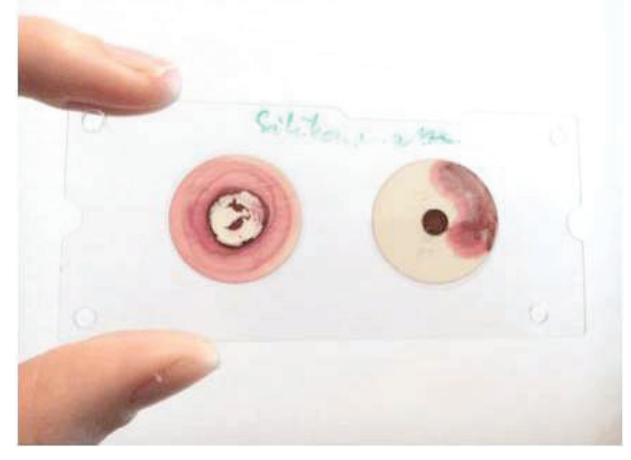

Abb. 3: Teststruktur bestehend aus Lasergeschnittenen Polycarbonat-Folien und einlaminierten $\mathrm{Al}_{2} \mathrm{O}_{3}$ Plättchen, gefärbt mit roter Indikatorflüssigkeit

Gut zu erkennen ist, dass die Fügeverbindung in der abgebildeten Teststruktur fluidisch nicht dicht ist. Dies resultiert aus an einer unzureichenden Verbindung zwischen Polycarbonat und Keramik. Eine Verbesserung der Fügeverbindung kann durch direkte Ablation der Keramik realisiert werden [26].

\section{Laserstrukturierung und Fügen}

Da bei der Lasermaterialbearbeitung keine mechanischen Werkzeuge zum Einsatz kommen, ist die Bearbeitung unabhängig von den mechanischen Materialeigenschaften des Werkstücks. Somit lassen sich auch schwierig zu bearbeitende Werkstoffe wie Keramiken abtragen. Zur Herstellung definierter Oberflächenstrukturen auf dem $\mathrm{Al}_{2} \mathrm{O}_{3}$-Substrat wurde eine Lasermikrostrukturierungsanlage (microSTRUCT ns, 3D-MICROMAC) mit einem UV-Kurzpulslaser (Avia NX 355-40, COHERENT) genutzt. Die Bearbeitungsanlage ist mit hochpräzisen Linearachsen sowie Galvanometerscannern ausgestattet und verfügt weiterhin über komplexe Messtechnik zur Analyse des Bearbeitungsergebnisses. Mit der vorhandenen Anlagentechnik können direktschreibend Strukturen bis unterhalb von $10 \mu \mathrm{m}$ reproduzierbar generiert werden. Für die ersten Untersuchungen wurden auf das $\mathrm{Al}_{2} \mathrm{O}_{3}$ Substrat Rechteckstrukturen mit $50 \mu \mathrm{m}$ bis $150 \mu \mathrm{m}$ Kantenlänge aufgebracht. Durch Variation der Prozessparameter wurden unterschiedliche Anordnungen, Tiefen und Abstände der Strukturen generiert. Anschließend wurden beide Fügepartner zusammengeführt, in einer Fügevorrichtung platziert und in eine Heißpresse überführt. Da wie in [26] beschrieben der Druck keinen Einfluss auf die Verbundfestigkeit hat wurde dieser bei $350 \mathrm{~N} / \mathrm{cm}^{2}$ konstant gehalten und die Temperatur von $145 \mathrm{C}$ bis $200^{\circ} \mathrm{C}$ variiert. Hierbei ist zu beachten, dass bei einem flächigen Temperatureintrag die Formstabilität des umgebenden Mikrofluidik-Systems nicht mehr gewährleistet ist. Daher wurde bei Temperaturen oberhalb der Glasübergangstemperatur des Polycarbonats die Sensorintegration separat vorgenommen. Dazu wurden die Substrate zunächst in die, sie einfassenden, Layer integriert und gefügt. Anschließend wurden die Fluidik- und Kontaktierungs-Layer bei Glasübergangstemperatur aufgebracht.

\section{Durchfluss-Fluidik}

Mit den zuvor ermittelten, optimierten Fügeparametern sowie der gezielten Aufrauhung des Sensor-Substrats konnte der beschriebene Sensor erfolgreich in einen Polycarbonat-Verbund einlaminiert werden. Im Anschluss wurde eine Durchfluss-Fluidik entwickelt in welche der Sensor integriert wurde um den Sauerstoffgehalt im Medium zu messen. In Abb. 4 ist eine Explosionsdarstellung des Chips zu sehen. Das System besteht aus einem Toplayer mit aufgeklebten Luer-Lock Anschlüssen, über die der 
Messkanal befüllt werden kann. In der Mitte befindet sich der Fluidiklayer mit der Messkammer und auf der Unterseite befindet sich der einlaminierte Sensor. Die Messkammer besitzt einen Durchmesser von $4.5 \mathrm{~mm}$ und eine Höhe von $0.5 \mathrm{~mm}$ und wird von einen $0.5 \mathrm{~mm}$ breiten und $0.25 \mathrm{~mm}$ hohen Kanal angeströmt. Der Medienwechsel erfolgte über einfache Spritzen mit Luer-Lock Anschluss.

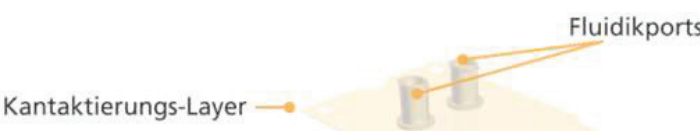

Abb. 4: Explosionsdarstellung der Durchfluss Fluidik für amperometrische Sauerstoffmessung

In Abb. 5 ist das mit Indikatorflüssigkeit befüllte mikrofluidische System zu sehen.

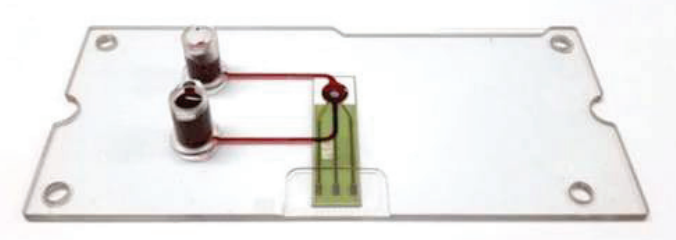

Abb. 5: Durchflussfluidik mit einlaminierten Sensor, gefüllt mit roter Lebensmittelfarbe zur Kanalvisualisierung.

\section{Amperometrische Sauerstoffmessung}

Der integrierte Dickschichtsensor besitzt keinen membrangetrennten Elektrolyten, vielmehr dient das Messgut selbst als Elektrolyt. Aus diesem Grund wurde eine 3molare $\mathrm{KCl}$-Lösung als Medium verwendet. Im ersten Schritt wurde das Voltammogram (Siehe Abb. 6) des Sensors aufgenommen um die ideale Polarisationsspannung $\mathrm{V}_{\mathrm{p}}$ für die anschließende amperometrische Messung zu ermitteln. Wie sich zeigt bildet sich zwischen 0.9 und $-0.4 \vee$ ein Plateau aus. Die Polarisationsspannung für die anschließenden amperometrischen Messungen wurde in die Mitte zwischen beide Werte auf $-0.7 \mathrm{~V}$ gelegt.

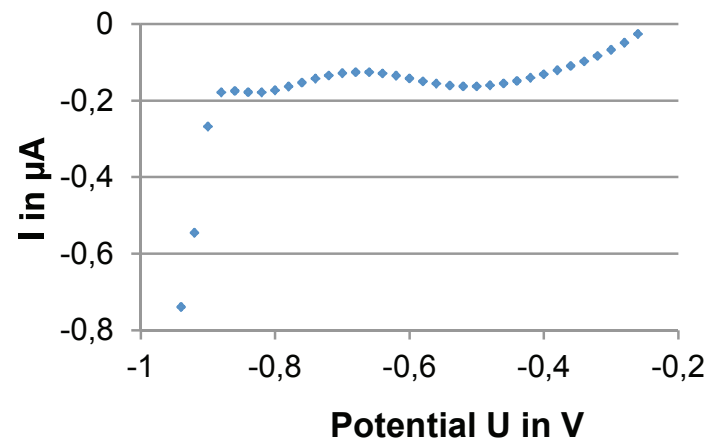

Abb. 6: Voltammogramm des Elektrolyten bei einer Scan-Rate von $0.02 \mathrm{~V}$ pro s.

Die Sauerstoffkonzentrationen kann eingestellt werden, indem verschiedene Lösungen in die Messkammer gepumpt werden und dann der Sauerstoffgehalt ohne Perfusion gemessen wird. Die drei Lösungen besaßen folgende Zusammensetzung:

1. Elektrolyt: $\mathrm{KCL}, 3 \mathrm{~mol} \mathrm{~L}^{-1}$.

2. $0.5 \mathrm{~mol} \mathrm{~L}^{-1}$ Natriumsulfit gelöst in $1 \mathrm{~mL}$ Elektrolyt.

3. $1 \mathrm{~mol} \mathrm{~L}^{-1}$ Natriumsulfit gelöst in $1 \mathrm{~mL}$ Elektrolyt.

Natriumsulfit bindet gelösten Sauerstoff im Wasser und wird aus diesem Grund auch als Korrosionsschutz verwendet [27]. Beim ersten Versuchsdurchlauf sollte der Anteil an gelöstem Sauerstoff der Sättigungskonzentration $c_{S, O 2}$ entsprechen. Diese wird gemäß Henry-Dalton-Gesetz mit dem Löslichkeitskoeffizient $\alpha$ wie folgt berechnet:

$c_{s, O 2}=\alpha \cdot p_{O 2}$

In der Luft beträgt der Sauerstoffpartialdruck $p_{O 2}$ auf Meeresniveau etwa $21.2 \mathrm{kPa}$. Bei einer Temperatur von $21^{\circ} \mathrm{C}$ sind dann etwa $8.7 \mathrm{mg}$ Sauerstoff in einem Liter Wasser gelöst. Eine entsprechend geringere Sauerstoffkonzentration ist unter Zugabe von Natriumsulfit zu erwarten. Da der elektrochemische Strom proportional zur Sauerstoffkonzentration im Elektrolyten ist müsste auch der gemessene Strom kleiner werden. In Abb. 7 sind die zeitlichen Verläufe des gemessenen Sensorstroms bei einer Polarisationsspannung $V_{p}$ von $-0.7 \mathrm{~V}$ für die drei Proben dargestellt. 


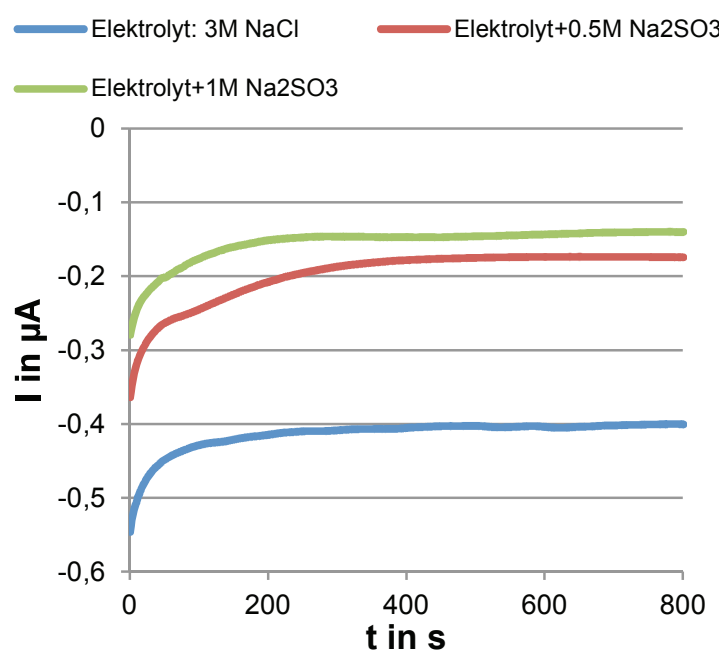

Abb. 7: Ergebnisse der amperometrischen Sauerstoffmessung für drei verschiedene Probenlösungen.

Gut zu erkennen ist der typische zeitliche Verlauf bei amperometrischen Messungen, mit einem Stromsprung wenn die Polarisationsspannung erreicht wird und einer langsamen Reduktion des Sensorstroms bis zu einem konstanten Endwert $I_{\text {end. }}$. Dieser Verlauf lässt sich durch die Sauerstoffverarmung in Folge der elektrochemischen Reaktion erklären und mathematisch mit der Cottrell-Gleichung beschreiben:

$$
I(t)=\frac{Z \cdot F \cdot A \cdot \sqrt{D}}{\sqrt{\pi \cdot t}} c_{O 2}+I_{0}=K \cdot c_{O 2}+I_{0}
$$

Die Gleichung enthält folgende Parameter:

\begin{tabular}{|c|c|}
\hline & Zahl der übertragenen Elektronen \\
\hline & Faraday-Konstante (96.486 As/mol) \\
\hline & Elektrodenoberfläche \\
\hline D & Diffusionskonstante Elektrolyt \\
\hline C & $\begin{array}{l}\text { Sauerstoffkonzentration zum Start der } \\
\text { Messung } \\
\text { Leckstrom bei Sauerstofffreiheit }\end{array}$ \\
\hline
\end{tabular}

Während der Messung ist der zusammengefasste Parameter K konstant, da sich sowohl der Elektrolyt als auch die Elektrodengeometrie nicht ändern. Somit kann die Sauerstoffkonzentration nach einer entsprechenden Kalibrierung direkt aus dem gemessenen Strom abgelesen werden [28]. Der Reststrom $\mathrm{I}_{0}$ wurde durch vollständigen Sauerstoffentzug mit einem Wert von $-0.14 \mu \mathrm{A}$ ermittelt. Daraus resultiert das $\mathrm{K}$ eine Größe von $-0.05 \mu \mathrm{Amg}^{-1} \mathrm{~L}$ hat und sich für die beiden Sauerstoffkonzentrationen $c_{1}$ und $c_{2}$, mit 0.5- und 1-molarer Natriumsulfit-Zugabe ein Wert von $4.8 \mathrm{mg} \mathrm{L}^{-1}$ und $3 \mathrm{mg} \mathrm{L}^{-1}$ bzw.
$34 \%$ und $55 \%$ bezogen auf die Sättigungskonzentration berechnet.

\section{Zusammenfassung und Ausblick}

Durch Optimierung der Fügeparameter sowie der gezielten Aufrauhung des Sensorsubstrats konnte erfolgreich ein kommerziell erhältlicher Dickfilm-Biosensor auf $\mathrm{Al}_{2} \mathrm{O}_{3}$-Substrat in das Multilagen-Zellkultivierungssystem einlaminiert werden. Im Anschluss erfolgten Sauerstoffmessungen in einer Durchfluss-Fluidik, wobei der Sauerstoffgehalt in der Lösung durch Zugabe von Natriumsulfit beeinflusst werden konnte. Während der Messung wurde das Medium selbst als Elektrolyt verwendet. Die aufgenommenen Kurven zeigten den für amperometrische Messungen typischen zeitlichen Verlauf. Über eine Kalibrierung ist so die Bestimmung der Sauerstoffkonzentration in der Lösung möglich.

In zukünftigen Arbeiten ist, wie bei anderen Biosensoren üblich, die Integration einer Membran erforderlich um Elektrolyt und Messgut voneinander zu trennen. Erste Vorarbeiten zum Einlaminieren von Polycarbonat-Membranen in das MultilagenSystem sind sehr vielversprechend. Gegebenenfalls kann auch die zur Fluidaktuierung verwendete Silikonmembran als Trennschicht genutzt werden. In diesem Fall wird zuerst der obere Teil mit integriertem Sensor und Elektrolytkammer gefügt. Dann erfolgt die Herstellung des fluidischen Teils. Im Anschluss werden beide Elemente mit dem beschriebenen nasschemischen Prozess und der Silikonmembran zusammengefügt. Der Elektrolyt kann dann durch eingebrachte Kanäle gewechselt werden, wenn dieser verbraucht ist.

Neben der beschriebenen amperometrischen Sauerstoffmessung ist auch eine TEERMessung sinnvoll, insbesondere wenn die Zelldichten von auf Membranen kultivierten Geweben wie z.B. Darmzellen von Interesse sind [7]. Dafür muss oberhalb der Membran eine Gegenelektrode, z.B. als Tauchelektrode realisiert werden. Weiterhin ist die Flussgeschwindigkeit in den Kanälen von großem Interesse, da diese Einerseits die Scherkraftbelastung von adhärenten Zellen wie Endothel festlegt [29] und Andererseits den Substanztransport im System maßgeblich beeinflusst. Neben der in, der Einleitung beschriebenen, $\mu$ PIV-Messung ist auch eine invasive Messung der Flussgeschwindigkeit mit dem kalorimetrischen Messprinzip möglich [30]. Der große Vorteil eines solchen Sensors ist die Möglichkeit die Flussgeschwindigkeit online zu messen und den Fluss ggf. durch Beeinflussung der integrierten Peristaltikpumpe zu beeinflussen Beide Sensoren können auch in Dickschichttechnik hergestellt 
werden, womit sie mit der beschriebenen Technologie integrierbar sind.

\section{Danksagung}

Die Autoren danken dem Freistaat Sachsen für die finanzielle Unterstützung im Rahmen des SAB-Projekts „UNILOC“ sowie dem BMWi und der AIF-Projekt $\mathrm{GmbH}$ für die Förderung des IGF-Projekts „Vaskularisierter Bioreaktor“.

\section{Literatur}

[1] A. Sinibaldi et al., J. Lightwave Technol. 2015, 33 (16), 3385.

[2] S. N. Bhatia, D. E. Ingber, Nat Biotechnol 2014, 32 (8), 760.

[3] A. L. Paguirigan, D. J. Beebe, Integr. Biol. 2009, 1 (2), 182.

[4] M. Baker, Nature 2011, 471 (7340), 661.

[5] A. Knight, Reviews on recent clinical trials 2008, 3 (2), 89.

[6] E.-M. Materne et al., JoVE 2015 (98).

[7] I. Maschmeyer et al., European Journal of Pharmaceutics and Biopharmaceutics 2015, 95, 77.

[8] B. Ataç et al., Lab Chip 2013, 13 (18), 3555.

[9] I. Maschmeyer et al., Lab Chip 2015, 15 (12), 2688.

[10] E. Schmälzlin et al., Biophysical Journal 2005, 89 (2), 1339.

[11] F. Sonntag et al., in SPIE LASE, SPIE Proceedings (Eds: U. Klotzbach, K. Washio, C. B. Arnold), SPIE 2015.

[12] N. Pereirarodriguez, Y. Sakai, J. Fujii, Sensors and Actuators B: Chemical 2008, 132 (2), 608.

[13] N. Ferrell et al., Biotechnol. Bioeng. 2010, 107 (4), 707.

[14] S. Richter, N. Qin, K. Pacak, G. Eisenhofer, Advances in pharmacology (San Diego, Calif.) 2013, 68, 285.

[15] Florian Schmieder et al., in Bionection 2015.

[16] H. K. Eltzschig, T. Eckle, Nature medicine 2011, 17 (11), 1391.

[17] M. Busek et al., in 18th ITI-Symposium 2015.

[18] V. Sunkara et al., Lab Chip 2011, 11 (5), 962.

[19] M. Busek et al., Biomedical Engineering / Biomedizinische Technik 2012, 57 (SI1 Track-E).

[20] M. Busek, M. Nötzel, C. Polk, F. Sonntag, J. Sens. Sens. Syst. 2013, 2 (2), 165.

[21] Mathias Busek et al., in Bionection 2015.

[22] C. Yi et al., Anal Bioanal Chem 2006, 384 (6), 1259.

[23] C. Galandival, TrAC Trends in Analytical Chemistry 1995, 14 (5), 225.

[24] S. Zhang, Biosensors and Bioelectronics 2000, 15 (5-6), 273.
[25] L. C. CLARK, Journal of applied physiology 1953, 6 (3), 189.

[26] V. Franke, F. Sonntag, G. Richter, U. Klotzbach, in 1st Electronic Systemintegration Technology 2006.

[27] H. Kerst, US 4278635 A.

[28] S. Herrmann et al., Sensors and Actuators B: Chemical 2000, 69 (1-2), 164.

[29] K. Schimek et al., Lab Chip 2013, 13 (18), 3588.

[30] M. Dijkstra et al., Sensors and Actuators A: Physical 2008, 143 (1), 1. 